Research Paper

\title{
Age- and Gender Dependent Association between Components of Metabolic Syndrome and Subclinical Arterial Stiifiness in a Chinese Population
}

\author{
Chunyan Weng ${ }^{1}$, Hong Yuan ${ }^{1,2}$, Xiaohong Tang ${ }^{1}$, Zhijun Huang ${ }^{1}$, Kan Yang', Wei Chen ${ }^{1}$, Pingting Yang ${ }^{3}$, Zhiheng \\ Chen ${ }^{3}$, Fangping $\mathrm{Chen}^{3}{ }^{凶}$ \\ 1. Department of Cardiology, the Third Xiangya Hospital, Central South University, Changsha 410013, China; \\ 2. Center of Clinical Pharmacology, the Third Xiangya Hospital, Central South University, Changsha 410013, China; \\ 3. Health Management Center, the Third Xiangya Hospital, Central South University, Changsha 410013, China.
}

$\triangle$ Corresponding author: Hong Yuan, PhD, Department of Cardiology, the Third Xiangya Hospital, Central South University, Changsha, 410013, China. Tel/Fax: +86-731-88618319 E-mail: hongyuanvip1@gmail.com

() Ivyspring International Publisher. This is an open-access article distributed under the terms of the Creative Commons License (http://creativecommons.org/ licenses/by-nc-nd/3.0/). Reproduction is permitted for personal, noncommercial use, provided that the article is in whole, unmodified, and properly cited.

Received: 2012.06.17; Accepted: 2012.10.12; Published: 2012.10.16

\begin{abstract}
Background: The aim of this study was to estimate the relationship between arterial stiffness and components of metabolic syndrome (MetS) in different age- and gender groups.

Methods: A total of 12,900 Chinese adults aged 20-79 years were recruited and stratified on the basis of gender and age. All participants underwent the measurement of waist circumference, blood pressure (BP), brachial-ankle pulse wave velocity (baPWV; an indicator of arterial stiffness), and blood chemistry. Multiple linear regression analysis was performed to evaluate the relationship between baPWV and above variables, to determine the relative influence of each component of MetS on baPWV.

Results: The prevalence of metabolic disorders except for low high-density lipoprotein cholesterol (HDL-C) was much higher in men than in women. All participants with MetS or any component of MetS except for low HDL-C had higher baPWV. BP was positively correlated with baPWV in all groups, while HDL-C was not correlated with baPWV in any groups. In addition, fasting glucose was related to baPWV in middle-aged adults and the elderly. Waist circumference had a positive association with baPWV in middle-aged adults and young men, triglyceride levels showed a significant correlation with baPWV in middle-aged women and young men. Of the MetS components, elevated BP was the strongest predictor of baPWV.

Conclusion: The prevalence of metabolic disorders and the association between baPWV and metabolic variables are dependent on age and gender. Different components of MetS exert distinct impacts on the baPWV in different age- and gender groups, with BP being the strongest predictor. It is suggested that age and gender should be taken into accounted in the management of MetS aiming to reduce subsequent complications.
\end{abstract}

Key words: Gender difference; Metabolic syndrome; Brachial-ankle pulse wave velocity; Subclinical arterial stiffness.

\section{INTRODUCTION}

Cardiovascular diseases (CVD) are major causes of morbidity and mortality worldwide. In 2011, the World Health Organization (WHO) reported that approximately 17.3 million people died of CVD, representing $30 \%$ of all global deaths, and the mortality was higher in white and black men than women. Most of the deaths from CVD occur in patients aged 75 years and older ${ }^{1}$. By 2030, it is estimated that there will be 23.6 million deaths from CVD. To identify individuals with high risk for CVD, the metabolic syn- 
drome (MetS), consisting of abdominal obesity, atherogenic dyslipidemia, hypertension, and hyperglycemia, has been developed. Although there is a debate on the concept of MetS, 2,3 it has been identified as an important and common cluster of risk factors of CVD by some organizations such as the International Diabetes Federation (IDF) ${ }^{4}$ and the World Health Organization(WHO). ${ }^{5}$ MetS is now commonly used in large-scale studies around the world. MetS often presents before the onset of CVD or diabetes, and the components of MetS are usually related to development and progression of CVD.

Atherosclerosis (AS) as an underlying cause of most CVD event, is usually identified before the presence of clinical manifestations of CVD. In clinical practice, pulse wave velocity (PWV) has been used to reflect the arterial stiffness and AS. A noninvasive measurement of brachial-ankle pulse wave velocity (baPWV), serving as a marker of AS with high reliability and validity, has been shown to correlate with the presence and extent of AS. ${ }^{6-9}$

It is important to investigate the association of MetS with AS at early stage which may guide to prevent or delay the development of AS. Studies examining the association between MetS and PWV have consistently shown that increased PWV in subjects with MetS or with larger number of MetS components. ${ }^{10-14}$ These suggest that the arterial stiffness in the MetS may be due to the subtle metabolic abnormalities, which develop gradually with aging. Although, the impact of age and gender on baPWV has been studied extensively, and age- and gender-dependent normograms have been proposed. ${ }^{15-17}$ However, the influence of age and gender dependent metabolic disorders on baPWV has not been well investigated. Furthermore, the relative importance of each component of MetS in different age and gender groups is still poorly understood. In the present study, a Chinese population (12,900 participants) was recruited and the age and gender dependent associations between baPWV and metabolism related variables were evaluated to determine the influence of each component of MetS on baPWV.

\section{MATERIALS AND METHODS}

\section{Study Population and Measurements}

The medical record of 12,900 participants who underwent a self-paid health examination in the Third Xiangya Hospital of Central South University was retrospectively analyzed from March 1, 2007 to October 31, 2008. Medical history was recorded and measurements of body weight, height, and waist circumference were done in all patients. Blood pressure
(BP) was measured in the right upper arm in the sitting position after a 10-15 minute rest between 7 AM and 9 AM using an Automatic Digital Blood Pressure Monitor. Body mass index (BMI) was calculated as body weight $(\mathrm{kg})$ divided by the square of body height $(\mathrm{m})$. Venous blood was collected in the morning after overnight fasting. The fasting glucose, total cholesterol, triglycerides, low-density lipoprotein cholesterol (LDL-C), and high-density lipoprotein cholesterol (HDL-C) were measured. The baPWV was measured in all subjects using the Colin-VP1000 (Komaki, Japan) between 8 AM and 10 AM by trained and experienced nurses. Measurement of baPWV was done according to previously described. ${ }^{8}$ In brief, the subject was asked to lie in a supine position for at least 5 mins. The cuffs were connected to a plethymographic sensor that determines volume pulse form and an oscillometric pressure sensor that measures BP. baPWV was calculated as follows: baPWV= (La/Lb)/Tba. ${ }^{17}$ This method has been validated previously. ${ }^{18}$

\section{Grouping and Definition of Metabolic Syn- drome}

Subjects younger than 20 years or older than 80 years were excluded from this study and subjects with drug treatment, CAD, stroke, renal insufficiency, peripheral arterial disease and history of smoking, had been excluded. All the subjects were divided into 3 age groups: young group (20-39 years), middle-aged group (40-59 years), and elderly group (60-79 years). MetS was defined in accordance with the criteria developed by International Diabetes Federation (IDF), 18 but a lower waist circumference cutoff for the Asian population was employed. ${ }^{19}$ The diagnosis criteria for MetS by IDF were as follows. a, required criteria: He or she must have central obesity (waist circumference: $\geq 90$ $\mathrm{cm}$ in men and $\geq 80 \mathrm{~cm}$ in women). b, plus any two of the following four factors: Elevated triglyceride level: $\geq 1.7 \mathrm{mmol} / \mathrm{L}$ (or $150 \mathrm{mg} / \mathrm{dL}$ ); Reduced HDL-C: < 40 $\mathrm{mg} / \mathrm{dL}$ (male) or $50 \mathrm{mg} / \mathrm{dL}$ (female); Elevated blood pressure $(\geq 130 / 85 \mathrm{~mm} \mathrm{Hg})$, and Fasting plasma glucose $\geq 5.6 \mathrm{mmol} / \mathrm{L}$ (or $100 \mathrm{mg} / \mathrm{dL}$ ). Or they had been diagnosed as dyslipidemia, Hypertension and Diabetes Mellitus with specific treatment for these abnormalities.

\section{Statistical Analysis}

Continuous variables were expressed as mean \pm standard deviation (SD) while categorical variables as proportions. One-way analysis of variance (ANOVA) followed by a post-hoc Student-Newman-Keuls test 
was for comparisons among multiple groups. The chi-square test-was used to compare the prevalence of MetS and MetS components across different age groups. Multiple linear regression model with stepwise selection was employed to estimate the association between 5 components of MetS and baPWV in each group stratified by gender and age. Standardized effect size ( $\beta$ value) was expressed to compare the relationship in each group. Statistical analyses were performed using SPSS version 17.0 (SPSS Inc., Chicago, IL, USA). A value of $\mathrm{P}<0.01$ was considered statistically significant.

\section{RESULTS}

\section{Demographic Data and Clinical Characteris- tics}

A total of 12,900 participants were recruited into this study, and $65.8 \%$ of them were men. The characteristics of these subjects are summarized in Table 1. The mean age of female and male subjects was $48.93 \pm 11.61$ years and $48.67 \pm 11.56$ years, respectively $(\mathrm{P}=0.243)$. The mean baPWV was $1420 \pm 315.8 \mathrm{~cm} / \mathrm{s}$ in women and $1470 \pm 285.9 \mathrm{~cm} / \mathrm{s}$ in men $(\mathrm{P}<0.001)$. All other variables were significantly higher in men than in women.

Table I. Demographics and clinical characteristics of subjects (mean $\pm S D$ ).

\begin{tabular}{llll}
\hline Variables & $\begin{array}{l}\text { Female } \\
(\text { mean } \pm S D) \\
(\mathrm{N}=4416)\end{array}$ & $\begin{array}{l}\text { Male } \\
(\text { mean } \pm \text { SD }) \\
(\mathrm{N}=8484)\end{array}$ & $P$ \\
\hline Age (year) & $48.93 \pm 11.61$ & $48.67 \pm 11.56$ & 0.243 \\
SBP $(\mathrm{mm} \mathrm{Hg})$ & $123 \pm 21.8$ & $127 \pm 18.1$ & $<0.001$ \\
DBP $(\mathrm{mm} \mathrm{Hg})$ & $74 \pm 12.0$ & $80 \pm 12.4$ & $<0.001$ \\
BMI $\left(\mathrm{kg} / \mathrm{m}^{2}\right)$ & $23.03 \pm 3.00$ & $25.01 \pm 3.07$ & $<0.001$ \\
Waist circumference $(\mathrm{cm})$ & $76.15 \pm 7.86$ & $86.81 \pm 8.32$ & $<0.001$ \\
Fasting glucose & $4.91 \pm 1.05$ & $5.15 \pm 1.47$ & $<0.001$ \\
(mmol/L) & $4.88 \pm 0.93$ & $4.98 \pm 0.97$ & $<0.001$ \\
TC $(\mathrm{mmol} / \mathrm{L})$ & $1.16 \pm 0.99$ & $2.20 \pm 0.85$ & $<0.001$ \\
TG $(\mathrm{mmol} / \mathrm{L})$ & $2.84 \pm 0.80$ & $2.89 \pm 0.85$ & 0.003 \\
LDL-C $(\mathrm{mmol} / \mathrm{L})$ & $1.42 \pm 0.32$ & $1.16 \pm 0.28$ & $<0.001$ \\
HDL-C $(\mathrm{mmol} / \mathrm{L})$ & $1420 \pm 315.8$ & $1470 \pm 285.9$ & $<0.001$ \\
baPWV $(\mathrm{cm} / \mathrm{s})$ & & & \\
\hline
\end{tabular}

Note: SD, standard deviation; SBP, systolic blood pressure; DBP, diastolic blood pressure; BMI, body mass index; TC, total cholesterol; TG, triglyceride; LDL-C, low-density lipoprotein cholesterol; HDL-C, high-density lipoprotein cholesterol; baPWV, brachial-ankle pulse wave velocity.

\section{Prevalence of Metabolic Syndrome and Each Metabolic Syndrome Component in Different Groups}

MetS was diagnosed in $19.4 \%$ of the participants (14.0\% in women and $22.1 \%$ in men, $\mathrm{P}<0.001)$. Except for HDL-C, the prevalence of other MetS components were significantly higher in men than women
$(\mathrm{P}<0.001)$ as shown in Table 2 . The prevalence of elevated $\mathrm{BP}$ and elevated fasting glucose increased with age irregardless of gender $(\mathrm{P}<0.001)$ and that of central obesity, elevated triglyceride, and MetS increased with age in women but not in men. In contrast, the prevalence of low HDL-C was not linearly correlated with age (Figure 1).

Table 2. Gender dependent prevalence of metabolic syndrome and metabolic syndrome components.

\begin{tabular}{llll}
\hline Variables & \multicolumn{2}{l}{ Prevalence $(\% / \mathrm{n})$} & $P$ \\
\cline { 2 - 3 } & Female $(\mathrm{n}=4416)$ & Male $(\mathrm{n}=8484)$ & \\
\hline MetS & $14.0(620)$ & $22.1(1877)$ & $<0.001$ \\
L-HDL & $15.2(670)$ & $15.1(1278)$ & 0.877 \\
Obesity & $32.9(1455)$ & $37.6(3189)$ & $<0.001$ \\
E-TG & $22.7(1003)$ & $48.6(4125)$ & $<0.001$ \\
E-FG & $12.6(557)$ & $18.4(1561)$ & $<0.001$ \\
E-BP & $37.4(1650)$ & $49.8(4228)$ & $<0.001$ \\
\hline
\end{tabular}

Note: MetS, metabolic syndrome; L-HDL, low high-density lipoprotein cholesterol; Obesity, central obesity; E-TG, elevated triglyceride; E-FG, elevated fasting glucose; E-BP, elevated blood pressure.

\section{Association of Metabolic Variables with baPWV in Different Groups}

The association of metabolic variables with baPWV is summarized in Table 3 . In general, the HDL-C level, LDL-C level and level of total cholesterol and triglyceride were not statistically associated with baPWV in any group. However, age, systolic BP and BMI were positively correlated with baPWV in groups. While diastolic BP was correlated with baPWV in young and middle-aged adults but not in the elderly. High baPWV was associated with waist circumference except in young men and associated with fasting glucose except in young women.

\section{Influence of Metabolic Syndrome Components on BaPWV in Different Groups}

Among the MetS components, the HDL-C level was not a predictor of elevated baPWV in all groups, while both systolic BP and diastolic BP were strongly positively associated with the elevated baPWV across all groups (Table 4). However, the remaining 3 components of MetS were associated with elevated baPWV regardless of gender, except for triglycerides in men. Fasting glucose level was positively correlated with elevated baPWV in middle-aged adults and the elderly. Waist circumference was significantly associated with the elevated baPWV in young and middle-aged adults except in young women. Triglyceride level was positively associated with baPWV in young men and middle-aged women. Among 5 components, BP had the strongest association with baPWV in all groups. 
Figure I. Age dependent prevalence of MetS and MetS components in different gender groups. L-HDL, low high-density lipoprotein cholesterol; Obesity, central obesity; TG, triglyceride; FG, fasting glucose; BP, blood pressure.
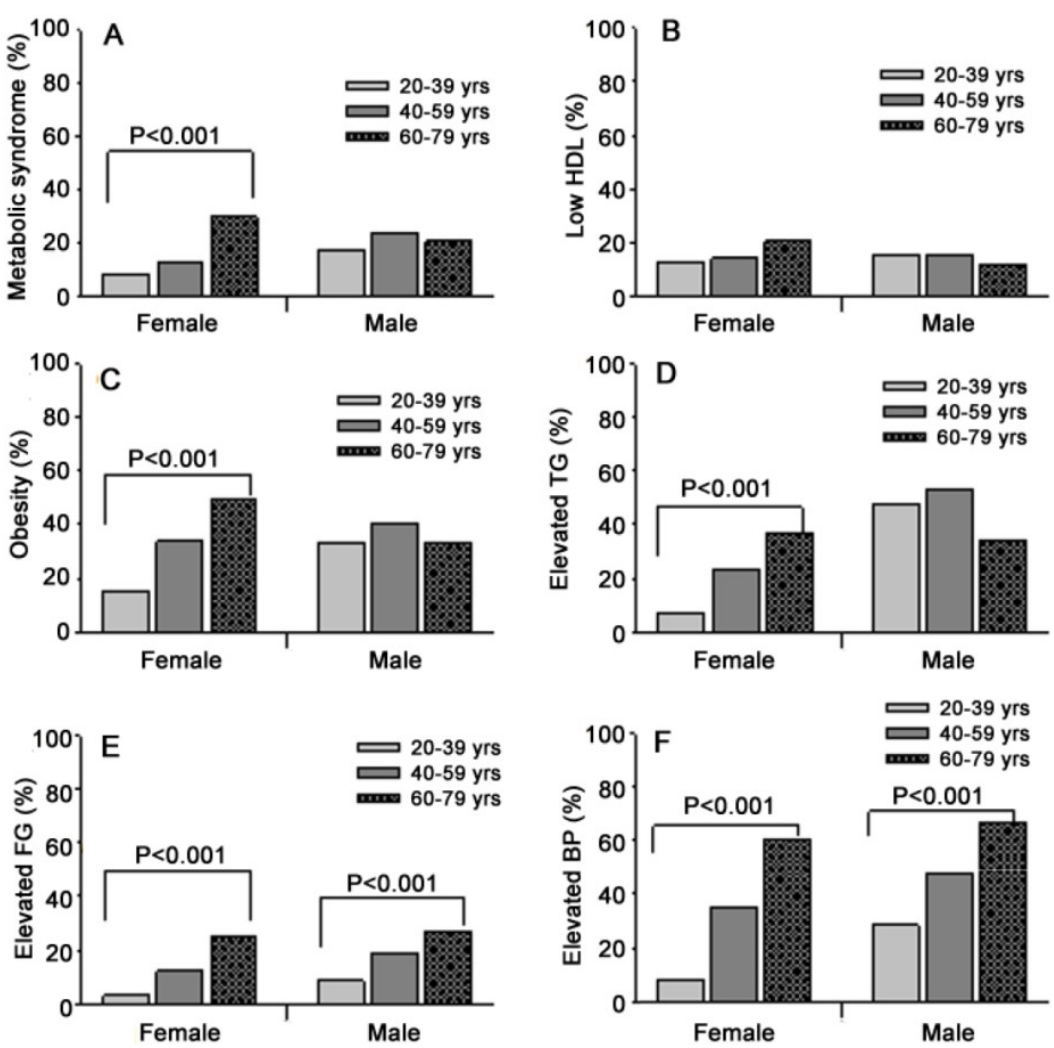

TABLE 3. Predictors of baPWV in different age groups and gender groups in multiple linear regression with stepwise selection.

\begin{tabular}{|c|c|c|c|c|c|c|c|c|}
\hline \multirow[t]{3}{*}{ Predictors } & \multicolumn{2}{|c|}{ Young $(n=2605)$} & \multicolumn{2}{|c|}{ Middle-aged $(n=7929)$} & \multicolumn{2}{|c|}{ Elderly $(n=2366)$} & \multicolumn{2}{|c|}{ Total $(n=12900)$} \\
\hline & $\mathrm{B}$ & & B & & B & & B & \\
\hline & $\begin{array}{l}\text { Female } \\
(\mathrm{n}=887)\end{array}$ & $\begin{array}{l}\text { Male } \\
(\mathrm{n}=1718)\end{array}$ & $\begin{array}{l}\text { Female } \\
(\mathrm{n}=2694)\end{array}$ & $\begin{array}{l}\text { Male } \\
(\mathrm{n}=5235)\end{array}$ & $\begin{array}{l}\text { Female } \\
(\mathrm{n}=835)\end{array}$ & $\begin{array}{l}\text { Male } \\
(n=1531)\end{array}$ & $\begin{array}{l}\text { Female } \\
(\mathrm{n}=4416)\end{array}$ & $\begin{array}{l}\text { Male } \\
(\mathrm{n}=8484)\end{array}$ \\
\hline SBP (mm Hg) & $3.93^{* *}$ & $2.23^{* *}$ & $6.48^{* *}$ & $5.62^{* *}$ & $8.88^{* *}$ & $8.59^{* *}$ & $8.14^{* *}$ & $7.42^{* *}$ \\
\hline DBP (mm Hg) & $2.91^{* *}$ & $4.02^{* *}$ & $1.25^{*}$ & $2.33^{* *}$ & & & $1.19^{* *}$ & \\
\hline BMI $\left(\mathrm{kg} / \mathrm{m}^{2}\right)$ & $10.29^{* *}$ & $4.96^{*}$ & $5.89^{*}$ & $10.59^{* *}$ & $20.39^{* *}$ & $32.78^{* *}$ & $13.47^{* *}$ & $16.13^{* *}$ \\
\hline $\mathrm{WC}(\mathrm{cm})$ & $2.87^{*}$ & & $2.4^{*}$ & $1.76^{*}$ & $5.69^{*}$ & $8.18^{* *}$ & $3.76^{* *}$ & $3.00^{* *}$ \\
\hline $\mathrm{FG}(\mathrm{mmol} / \mathrm{L})$ & & $9.05^{*}$ & $24.48^{* *}$ & $10.31^{* *}$ & $28.57^{* *}$ & $28.57^{* *}$ & $25.97^{* *}$ & $12.49^{* *}$ \\
\hline
\end{tabular}

Note: $\beta$, unstandardized regression coefficient; SBP, systolic blood pressure; DBP, diastolic blood pressure; BMI, body mass index; WC, waist circumference; FG, fasting glucose. HDL-C, LDL-C, total cholesterol and triglyceride were not statistically significant in all regression analyses. There were discrepancies in the number of subjects in each age group because all analyses relied on subjects with complete information on the variables chosen in the stepwise regression analyses. ${ }^{*} P<0.01,{ }^{* *} P<0.001$

Table 4. Components of metabolic syndrome as predictors of baPWV in different age groups and gender groups in multiple linear regression with stepwise selection.

\begin{tabular}{|c|c|c|c|c|c|c|c|c|}
\hline \multirow[t]{3}{*}{ Predictors } & \multicolumn{2}{|c|}{ Young (n=2605) } & \multicolumn{2}{|c|}{ Middle-aged (n=7929) } & \multicolumn{2}{|c|}{ Elderly (n=2366) } & \multicolumn{2}{|c|}{ Total $(n=12900)$} \\
\hline & \multicolumn{2}{|c|}{$\beta$ (effect size) } & \multicolumn{2}{|c|}{$\beta$ (effect size) } & \multicolumn{2}{|c|}{$\beta$ (effect size) } & \multicolumn{2}{|c|}{$\beta$ (effect size) } \\
\hline & $\begin{array}{l}\text { Female } \\
(\mathrm{n}=887)\end{array}$ & $\begin{array}{l}\text { Male } \\
(\mathrm{n}=1718)\end{array}$ & $\begin{array}{l}\text { Female } \\
(\mathrm{n}=2694)\end{array}$ & $\begin{array}{l}\text { Male } \\
(n=5235)\end{array}$ & $\begin{array}{l}\text { Female } \\
(\mathrm{n}=835)\end{array}$ & $\begin{array}{l}\text { Male } \\
(\mathrm{n}=1531)\end{array}$ & $\begin{array}{l}\text { Female } \\
(\mathrm{n}=4416)\end{array}$ & $\begin{array}{l}\text { Male } \\
(\mathrm{n}=8484)\end{array}$ \\
\hline \multicolumn{9}{|c|}{ HDL-C (mmol/L) } \\
\hline WC (cm) & & $0.09^{* *}$ & $0.05^{*}$ & $0.07^{* *}$ & & & $0.05^{* *}$ & $0.08^{* *}$ \\
\hline $\mathrm{TG}(\mathrm{mmol} / \mathrm{L})$ & & $0.08^{*}$ & $0.06^{* *}$ & & & & $0.05^{* *}$ & \\
\hline $\mathrm{FG}(\mathrm{mmol} / \mathrm{L})$ & & & $0.11^{* *}$ & $0.09^{* *}$ & $0.14^{* *}$ & $0.11^{* *}$ & $0.11^{* *}$ & $0.11^{* *}$ \\
\hline SBP (mm Hg) & $0.32^{* *}$ & $0.15^{* *}$ & $0.66^{* *}$ & $0.51^{* *}$ & $0.61^{* *}$ & $0.56^{* *}$ & $0.81^{* *}$ & $0.71^{* *}$ \\
\hline DBP (mm Hg) & $0.23^{* *}$ & $0.38^{* *}$ & $0.02^{*}$ & $0.10^{* *}$ & $0.12^{*}$ & $0.08^{*}$ & $0.18^{* *}$ & $0.14^{* *}$ \\
\hline
\end{tabular}

Note: $\beta$, standardized regression coefficient; WC, waist circumference; TG, triglyceride; HDL-C, high-density lipoprotein cholesterol; SBP, systolic blood pressure; DBP, diastolic blood pressure; FG, fasting glucose. e: There were discrepancies in the number of subjects in each age group because all analyses relied on subjects with complete information on the variables chosen in the stepwise regression analyses. ${ }^{*} P<0.01,{ }^{, *} P<0.001$. 


\section{Influence of Metabolic Syndrome and Meta- bolic Syndrome Components on BaPWV in Different Gender Groups}

The baPWV was significantly higher in subjects with MetS, elevated BP or elevated fasting glucose in both men and women. There was no significant difference in baPWV between subjects with reduced
HDL-C and normal HDL-C. Women with central obesity or elevated triglyceride had significantly higher baPWV while there was no significance in men. When compared with men, women with MetS or MetS components had higher baPWV $(\mathrm{P}<0.001)$, except in subjects with low HDL-C (Figure 2).
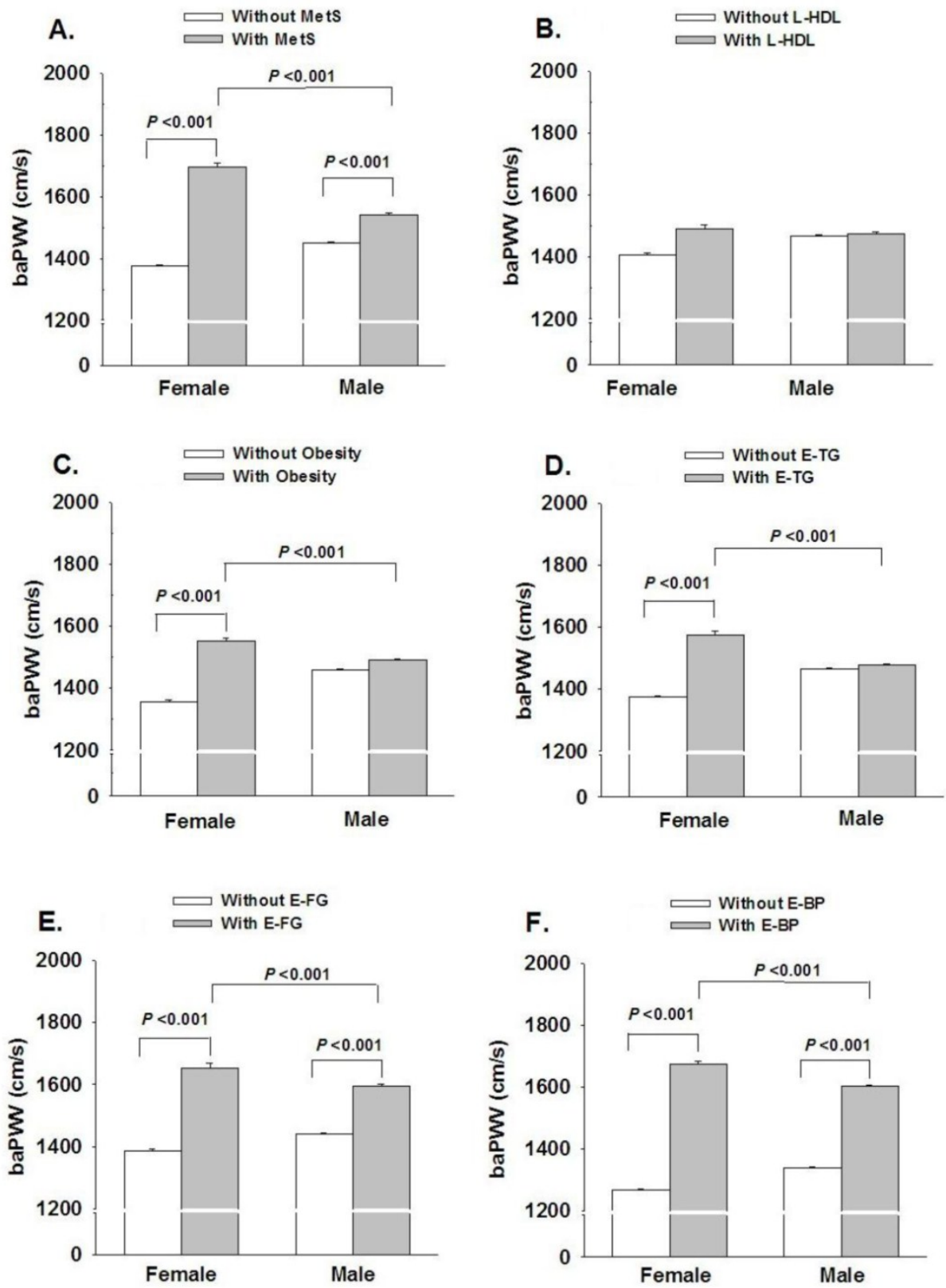

Figure 2. Gender dependent influence of MetS and MetS components on baPWV. L-HDL, low high-density lipoprotein cholesterol; Obesity, central obesity; E-TG, elevated triglyceride; E-FG, elevated fasting glucose; E-BP, elevated blood pressure. 


\section{Influence of Each Component of Metabolic Syndrome on baPWV in Different Gender Groups}

Subjects without MetS and MetS components served as controls. The baPWV in subjects with low HDL-C was comparable to that in controls regardless of genders. The baPWV in women with central obesity, elevated triglyceride or elevated fasting glucose was significantly higher than that in corresponding controls (Figure 3). In contrast, the baPWV in men with central obesity and elevated triglyceride groups was similar to that in the control group, but baPWV in subjects with elevated fasting glucose was higher than that in controls. The baPWV in the subjects with elevated $\mathrm{BP}$ was the highest in all groups $(\mathrm{P}<0.001)$.

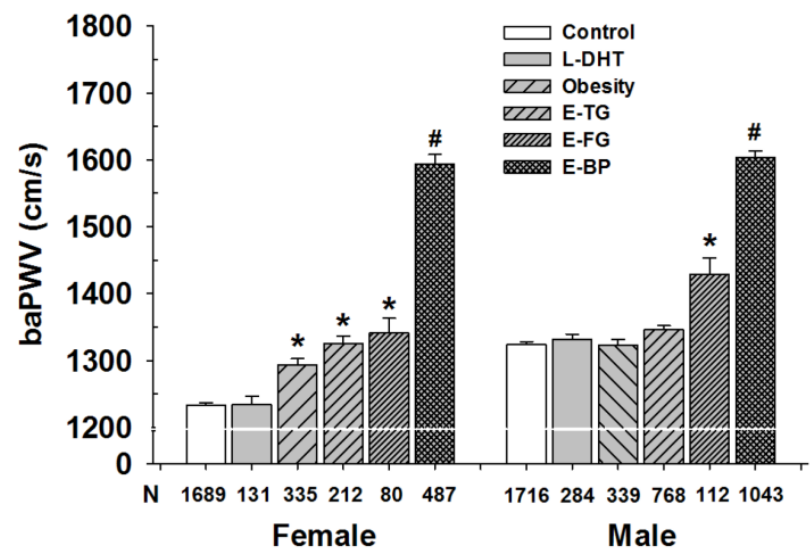

Figure 3. Influence of each MetS component on baPWV in different gender groups. L-HDL, low high-density lipoprotein cholesterol; Obesity, central obesity; E-TG, elevated triglycerides; E-FG, elevated fasting glucose; E-BP, elevated blood pressure. Control: subjects without metabolic disorders. Note: there was not difference between L-HDL group and control in men and women; The baPWV in subjects with obesity, E-TG or E-FG in women was comparable; L-HDL, Obesity and E-TG groups in women were not statistically significant each other. ${ }^{*} P<0.00$ I vs. control; $\# P<0.00$ I vs. E-FG group.

\section{Influence of Number of Metabolic Syndrome Components on BaPWV in Different Gender Groups}

Figure 4 shows the mean baPWV according to the number of MetS components after adjustment for age. The mean baPWV in women with $0,1,2,3$ and $\geq 4$ components of MetS was 1,233, 1,413, 1,574, 1707 and $1,814 \mathrm{~cm} / \mathrm{s}$, respectively $(\mathrm{P}<0.001)$, and that in men was 1,324, 1,450, 1,502, 1,574, and $1,628 \mathrm{~cm} / \mathrm{s}$ $(\mathrm{P}<0.001)$.

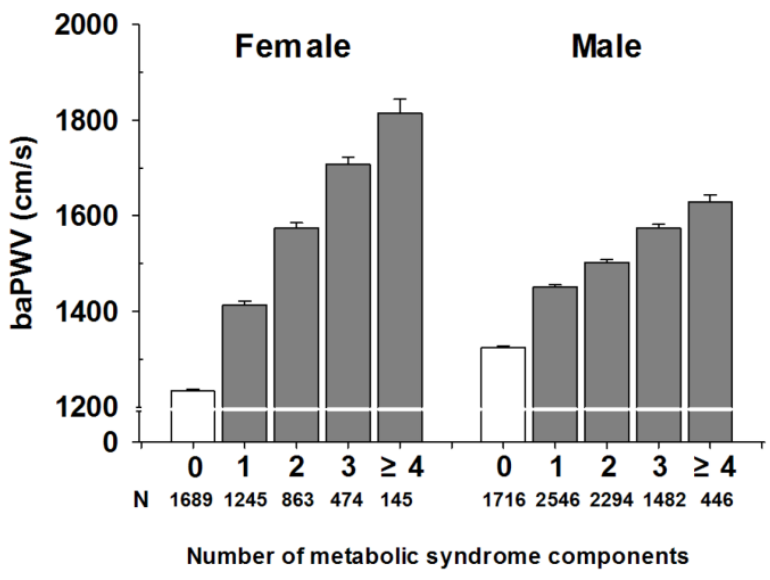

Figure 4. Influence of number of MetS components on baPWV in mean and women. $P<0.001$.

\section{DISCUSSION}

Our results showed that, although the MetS is linked to elevated baPWV, different metabolic variables and each component of MetS have distinct impacts on baPWV in age and gender dependent manner. The prevalence of MetS and MetS components, except for low HDL-C, was higher in men than in women, suggesting the gender dependent manner.

These results were similar to those in studies of other countries. 19,20 Furthermore, the prevalence of MetS, elevated BP and elevated fasting glucose increased with age in both gender groups, that of obesity and elevated triglyceride increased with age in women but not in men, and that of low HDL-C was not related to the gender and age. These results suggest that the prevalence of MetS and MetS components are age- and gender dependent, and each MetS component has a distinct association with age and gender. The age and gender dependent prevalence of MetS components suggest that management of metabolic disorders should take the age and gender into accounted.

Most previous studies on the association between metabolic variables and baPWV were conducted in either the general populations or specific subgroups. ${ }^{10,21,22}$ Furthermore, studies on MetS only reported the association with age. In the present study, a large number of participants were recruited and evaluation of the association was done in different age groups and gender groups. The large sample size allowed us to employ the multivariable analysis to determine the independent influence of each component of MetS on the baPWV. To avoid false positive results, appropriate statistical methods were used to adjust for multiple comparisons. 
Age and gender have long been considered as important factors affecting the baPWV. ${ }^{17}$ In this study, the influence of MetS and MetS components on the baPWV was evaluated in different age groups and gender groups. All subjects with MetS or any component of MetS except for low HDL-C had higher baPWV, and women with metabolic disorders had higher baPWV than men did, but the specific mechanism is required to be elucidated further. Systolic BP and diastolic BP were positively associated with baPWV in all groups, whereas fasting glucose was positively associated with baPWV only in the middle-aged adults and the elderly, not in young adults. Waist circumference was positively associated with baPWV in middle-aged adults and young men, triglyceride level showed a significant association with baPWV in middle-aged women and young men, but HDL-C was not associated with baPWV in any group.

Obesity, a result of positive energy balance, especially the excess visceral adiposity, leads to insulin resistance and provides an adverse metabolic environment that facilitates the development of MetS and CVD. ${ }^{23,24}$ The prevalence of obesity is strongly related to life expectancy: the higher the prevalence of obesity, the shorter the life expectancy in a country. ${ }^{25}$ In addition, the prevalence of obesity is gender dependent. In our study, the prevalence of obesity was significantly higher in men than in women $37.6 \%$ and $32.9 \%$, respectively), which is consistent findings in other countries. 26 Interestingly, in different age groups, the prevalence of obesity increased with age in women but not in men. This may partially attribute to the effect of estrogen on lipid metabolism. ${ }^{27}$ In a large cohort study in the United States, women with a BMI of $\geq 35 \mathrm{~kg} / \mathrm{m}^{2}$ had an odds ratio (OR) of 2.7 for coronary arterial disease (CAD), an OR of 5.4 for hypertension and an OR of 12.5 for diabetes, while the OR in men was 1.7 for CAD, 5.6 for hypertension and 8.3 for diabetes. ${ }^{28}$ In addition, the baPWV, an early marker for AS and artery stiffness, was determined in our study and results showed that obese women had higher baPWV than those without obesity, which, however, was not found in men. In addition, the baPWV in women was significantly higher than in men, which was consistent with the findings in clinical practice. The age and gender dependent prevalence and influence of triglyceride on baPWV were similar to those of obesity, which may attribute to the fact that excess triglyceride leads to obesity. ${ }^{29}$ Correlation analysis revealed triglyceride level was not correlated with baPWV, and the influence of triglyceride on the baPWV seems to be relevant with the influence of obesity.

HDL-C is a strong inverse predictor of cardio- vascular events. Numerous interventional studies in experimental AS have shown the potential for HDL-C elevation to modify plaque favorably. ${ }^{30,31}$ Feig et al. ${ }^{32}$ also showed that HDL-C promotes the AS regression in mice. However, in our study, HDL-C level was not correlated with baPWV. There were some limitations for the HDL-C measurement. The compositional complexity and functional role of HDL-C were not taken into account. Khera et al. ${ }^{33}$ showed that the cholesterol efflux capacity from macrophages, a metric of HDL-C function, had a strong inverse association with both carotid intima-media thickness and the likelihood of angiographic CAD, independent of the HDL-C level. Furthermore, the influence of HDL-C on the inflammation, reverse cholesterol transport, oxidation, endothelial function, and gene transcription can not be assessed by the HDL-C level. In future studies, we will examine the compositions and function of HDL-C.

In addition, the influence of MetS components on baPWV was also assessed in our study. In women, the baPWV in subjects with low HDL-C was comparable to that in controls; however, in obese women with elevated triglyceride and elevated fasting glucose, the baPWV was significantly higher than that in the control group, and they were not significant from each other. In subjects with elevated fasting glucose, baPWV was significantly higher than that in controls. In both women and men, elevated BP had the highest baPWV in all groups. Therefore, in women, the order in which MetS components affect the baPWV is as follow: elevated $\mathrm{BP}>$ elevated fasting glucose $=$ elevated triglyceride $=$ obesity $>$ low HDL-C $=$ control. While in men, the order is as follows: elevated BP > elevated fasting glucose $>$ elevated triglyceride $=$ obesity $=$ low HDL-C $=$ control. Among the MetS components, elevated $\mathrm{BP}$ is the strongest predictor of baPWV. Obesity is essential for the MetS and may lead to other metabolic disorders. ${ }^{34}$ Lipid disorders can increase the sympathetic activity via the adipocyte pathways, which results in elevation of BP. ${ }^{35}$ Although the elevated BP significantly affects the arterial stiffness, when combined with other metabolic disorders, the vascular dysfunction becomes a more significant factor ${ }^{36}$ and the baPWV increases with addition of any metabolic disorder, which is consistent with results from a study of Nakanishi et al ${ }^{10}$. BP lowering has been the most important intervention to prevent CVD and cardiovascular events. However, it is also necessary to manage other metabolic disorders as much as possible.

In conclusion, the prevalence of MetS and MetS components is age and gender dependent, and elevated baPWV is associated with different metabolic 
variables, including waist circumference in middle-aged adults and young men, fasting blood glucose in middle-aged adults and the elderly, and BP in most subjects. Each component of MetS has a distinct impact on baPWV in age and gender dependent manners, and the elevated BP $(\geq 130 / 85 \mathrm{mmHg})$ is the strongest predictor of arterial stiffness. These results suggest that age and gender should be taken into accounted in the management of MetS to reduce subsequent complications. However, there are limitations of baPWV. When the PWV values are high, confounding factors such as blood pressure, blood flow, and sympathetic tone might increase the variability of PWV. Even though an augmented variability of baPWV should be taken into account in cases of elevated baPWV, the reproducibility (less than 20\%) of the baPWV measurement is acceptable in clinical practice.

\section{ACKNOWLEDGEMENTS}

We thank Dr. Zhu for the help of manuscript preparation.

\section{Competing Interests}

The authors have declared that no competing interest exists.

\section{References}

1. Lloyd-Jones D, Adams R, Carnethon M, et al. Heart disease and stroke statistics--2009 update: a report from the American Heart Association Statistics Committee and Stroke Statistics Subcommittee. Circulation. 2009; 119: e21-181.

2. Gale EA. The myth of the metabolic syndrome. Diabetologia. 2005; 48 : 1679-83.

3. Kahn R, Buse J, Ferrannini E, et al. The metabolic syndrome: time for a critical appraisal. Joint statement from the American Diabetes Association and the European Association for the Study of Diabetes. Diabetologia. 2005; 48: 1684-99.

4. Alberti KG, Zimmet P, Shaw J, et al. The metabolic syndrome--a new worldwide definition. Lancet. 2005; 366: 1059-62.

5. Alberti KG, Zimmet PZ. Definition, diagnosis and classification of diabetes mellitus and its complications. Part 1: diagnosis and classification of diabetes mellitus provisional report of a WHO consultation. Diabet Med. 1998; 15: 539-53.

6. Mack WJ, LaBree L, Liu C, et al. Correlations between measures of atherosclerosis change using carotid ultrasonography and coronary angiography. Atherosclerosis. 2000; 150: 371-9.

7. Teragawa H, Kato M, Kurokawa J, et al. Usefulness of flow-mediated dilation of the brachial artery and/or the intima-media thickness of the carotid artery in predicting coronary narrowing in patients suspected of having coronary artery disease. Am J Cardiol. 2001; 88: 1147-51.

8. Yamashina A, Tomiyama H, Takeda K, et al. Validity, reproducibility, and clinical significance of noninvasive brachial-ankle pulse wave velocity measurement. Hypertens Res. 2002; 25: 359-64.

9. Lorenz MW, Markus HS, Bots ML, et al. Prediction of clinical cardiovascular events with carotid intima-media thickness: a systematic review and meta-analysis. Circulation. 2007; 115: 459-67.

10. Nakanishi N, Shiraishi T, Wada M. Brachial-ankle pulse wave velocity and metabolic syndrome in a Japanese population: the Minoh study. Hypertens Res. 2005; 28: 125-31.

11. Henry RM, Ferreira I, Dekker JM, et al. The metabolic syndrome in elderly individuals is associated with greater muscular, but not elastic arterial stiffness, independent of low-grade inflammation, endothelial dysfunction or insulin resistance--The Hoorn Study. J Hum Hypertens. 2009; 23: 718-27.
12. Nam JS, Park JS, Cho MH, et al. The association between pulse wave velocity and metabolic syndrome and adiponectin in patients with impaired fasting glucose: cardiovascular risks and adiponectin in IFG. Diabetes Res Clin Pract 2009;84:145-51.

13. Satoh H, Kishi R, Tsutsui $H$. Metabolic syndrome is a significant and independent risk factor for increased arterial stiffness in Japanese subjects. Hypertens Res. 2009; 32: 1067-71.

14. Hirashiki A, Murohara T. The impact of pulse wave velocity in a Japanese population with metabolic syndrome. Hypertens Res. 2009; 32: 1045-46.

15. Li S, Chen W, Srinivasan SR, et al. Influence of metabolic syndrome on arterial stiffness and its age-related change in young adults: the Bogalusa Heart Study. Atherosclerosis. 2005; 180: 349-54.

16. De Angelis L, Millasseau SC, Smith A, et al. Sex differences in age-related stiffening of the aorta in subjects with type 2 diabetes. Hypertension. 2004; 44: 67-71.

17. Tomiyama H, Yamashina A, Arai T, et al. Influences of age and gender on results of noninvasive brachial-ankle pulse wave velocity measurement--a survey of 12517 subjects. Atherosclerosis. 2003; 166: 303-9.

18. Zimmet P, M M Alberti KG, Serrano Ríos M. A new international diabetes federation worldwide definition of the metabolic syndrome: the rationale and the results. Rev Esp Cardiol 2005;58:1371-6.

19. Scuteri A, Najjar SS, Orru' M, et al. Age- and gender-specific awareness, treatment, and control of cardiovascular risk factors and subclinical vascular lesions in a founder population: the SardiNIA Study. Nutr Metab Cardiovasc Dis. 2009; 19: 532-41.

20. Ford ES, Giles WH, Dietz WH. Prevalence of the metabolic syndrome among US adults: findings from the third National Health and Nutrition Examination Survey. JAMA. 2002; 287: 356-9..

21. Miyai N, Arita M, Miyashita K, et al. The influence of obesity and metabolic risk variables on brachial-ankle pulse wave velocity in healthy adolescents. J Hum Hypertens. 2009; 23: 444-50.

22. Choi KM, Lee KW, Seo JA, et al. Relationship between brachial-ankle pulse wave velocity and cardiovascular risk factors of the metabolic syndrome. Diabetes Res Clin Pract. 2004; 66: 57-61.

23. Després JP, Lemieux I, Bergeron J, et al. Abdominal obesity and the metabolic syndrome: contribution to global cardiometabolic risk. Arterioscler Thromb Vasc Biol. 2008; 28: 1039-49.

24. Viviance Z. Rocha and Peter Libby. Obesity, inflammation, atherosclerosis. Nat Rev Cardiol. 2009; 6: 399-409.

25. Preston $\mathrm{SH}$, Stokes A. Contribution of obesity to international differences in life expectancy. Am J Public Health. 2011; 101: 2137-43.

26. Abdullah A, Wolfe R, Stoelwinder JU, et al. The number of years lived with obesity and the risk of all-cause and cause-specific mortality. Int J Epidemiol. 2011; 40: 985-96.

27. Rossouw JE. Hormones, genetic factors, and gender differences in cardiovascular disease. Cardiovasc Res. 2002; 53: 550-7.

28. Patterson RE, Frank LL, Kristal AR, et al. A comprehensive examination of health conditions associated with obesity in older adults. Am J Prev Med. 2004; 27: 385-90.

29. Forouhi NG, Jenkinson G, Thomas EL, et al. Relation of triglyceride stores in skeletal muscle cells to central obesity and insulin sensitivity in European and South Asian men. Diabetologia. 1999; 42: 932-5.

30. Badimon JJ, Badimon L, Fuster V. Regression of atherosclerotic lesions by high density lipoprotein plasma fraction in the cholesterol-fed rabbit. J Clin Invest. 1990; 85: 1234-41.

31. Choudhury RP, Rong JX, Trogan E, et al. High-density lipoproteins retard the progression of atherosclerosis and favorably remodel lesions without sup- pressing indices of inflammation or oxidation. Arterioscler Thromb Vasc Biol. 2004; 24: 1904-9.

32. Feig JE, Rong JX, Shamir R, et al. HDL promotes rapid atherosclerosis regression in mice and alters inflammatory properties of plaque monocyte- derived cells. Proc Natl Acad Sci USA. 2011; 108: 7166-71.

33. Khera AV, Cuchel M, de la Llera-Moya M, et al. Cholesterol efflux capacity, high-density lipoprotein function, and atherosclerosis. $\mathrm{N}$ Engl J Med. 2011; 364: 127-35.

34. Després JP, Lemieux I. Abdominal obesity and metabolic syndrome. Nature 2006;444:881-7.

35. Scherer T, Buettner C. Yin and Yang of hypothalamic insulin and leptin signaling in regulating white adiposetissue metabolism. Rev Endocr Metab Disord. 2011; 12: 235-43.

36. Rambaran C, Chowienczyk P, Ritter J, et al. The vascular effects of metabolic impairment clusters in subjects of different ethnicities. Atherosclerosis. 2007; 192: 354-62. 